\title{
Publisher Correction: Two views of the cognitive brain
}

\section{David L. Barack and John W. Krakauer}

Correction to: Nature Reviews Neuroscience https://doi.org/10.1038/s41583-021-00448-6, published online 15 April 2021.

In the original article, the titles for references 44 and 87 were omitted and the journal name in reference 87 was spelt incorrectly. These references have now been corrected in the HTML and PDF versions of the manuscript.

https://doi.org/10.1038/s41583-021-00487-z I Published online 17 June 2021

(๑) Springer Nature Limited 2021 